\title{
Usefulness of Retina Codes in Biometrics ${ }^{\star}$
}

\author{
Thomas Fuhrmann, Jutta Hämmerle-Uhl, and Andreas Uhl \\ Department of Computer Sciences, Salzburg University, Austria \\ uhl@cosy.sbg.ac.at
}

\begin{abstract}
We discuss methods for generating retina codes from retinal images for biometric user authentication. Starting from the optical disc, concentric circles are placed over the binary vessel image for data sampling and different variants of retina code are generated after transformation to polar coordinates. The methods inter personal variability and robustness is evaluated on the publicly available DRIVE database. Results indicate a low inter personal variability questioning the usefulness of retina codes in sensible authentication systems.
\end{abstract}

\section{Introduction}

With the increasing usage of biometric systems the interest in not-yet widely accepted modalities rises. Retina features are among these potentially promising but not mainstream techniques. Being transparent, the retina is situated in the innermost part of the ocular fundus, retinal features mainly consist of blood vessels originating from the entry point of the optic nerve and spreading across the ocular fundus (see Fig. 1, a for an example). The pattern of these vessels is said to be unique for each individual person and might therefore be used for biometric recognition systems. However, the scanning operation is required to be much more intrusive and controlled as compared to e.g. iris-based systems due to the location in the inner parts of the eye and user acceptance of such conditions is generally low. Therefore, the primary application context of this modality will be in high security environments like military or governmental agencies.

According to literature [4, p.106ff], retina-scan based biometric systems exhibit the following strengths: high spoofing resistance, high stability in the sense of time-invariance, and high recognition accuracy.

In fact, due to their location at the background of the eye, retinal features can hardly be replaced or modified, also sensors capturing the respective images can hardly by fooled. On the other hand, the stability of retinal vessels is questionable since many eye diseases include some blood vessel pathology as found e.g. in proliferative diabetic retinopathy [3], which is characterized by new vessel growth especially near the optical disk. The possible impact of such diseases on retina feature based biometric systems has to be seriously considered and investigated before a sensible deployment should take place.

^ This work has been partially supported by the Austrian Science Fund, project no. L554-N15.

T. Wada, F. Huang, and S. Lin (Eds.): PSIVT 2009, LNCS 5414, pp. 624 632, 2009.

(C) Springer-Verlag Berlin Heidelberg 2009 
Eye-based biometric modalities in general are believed to be highly secure due to the well investigated low FAR of some popular iris recognition systems 5. However, also in iris recognition several techniques exist which exhibit significantly inferior recognition performance (e.g., based on histograms [6]). Obviously, it is not only the potential distinctiveness of the physiological trait that determines the recognition accuracy but of course the type of extracted template data plays an at least equally important role as well.

There is not much work available on using retinal features for biometric purposes. Most of the literature on retinal features is found in ophthalmology where retinal vessels are used in diagnosis or as landmarks for image registration (see e.g. (7)). Strengths and weaknesses of retina-scan based biometrics are discussed in [4, but no concrete feature extraction technique or template data structure is described. Crossings of retinal vessels are suggested to be used as biometric features in [8] and good accuracy is reported. Retica Systems Inc 11 offers a different (commercial) solution based on a "retina code" (inspired by Daugmans' "iris code" principle [5]).

In this work we discuss the use of retina codes for biometric recognition as inspired by the solution sketched by Retica Systems Inc. Section 2 discusses feature extraction techniques for subsequent retina code generation which include optical disc segmentation and retinal vessel extraction. Section 3 describes several variants of retina code generation and Section 4 is devoted to experimental testing with emphasis on the overall code variation within a population and FMR under signal distortion. Section 5 concludes the paper.

\section{Feature Extraction}

Retina-based methods use ocular fundus images as a source for extracting biometric features for user authentication. Extracting usable feature sets for retina code generation from these images requires a combination of two different preprocessing methods.

\subsection{Optical Disc Segmentation}

For retina code generation a reference point has to be defined (analogous to the center of the pupil with iris recognition). Here the optical disc (where the optical nerve leaves the retina) seems suitable. In the retinal images the optical disc appears as a bright circular shaped object partly covered with vessels that has a higher background luminance and higher local variance as the rest of the retina. So the center of the optical disc can be used as a reference point for developing a retina template out of a segmented vessel feature image.

Detection of the optical disc has been largely covered in literature and numerous methods have been developed. Many applications choose a method that uses circular Hough transform on an thresholded edge image of the retinal surface as in Barret et al. [10. In our context this method sometimes yields poor

1 wWw.retica. com 


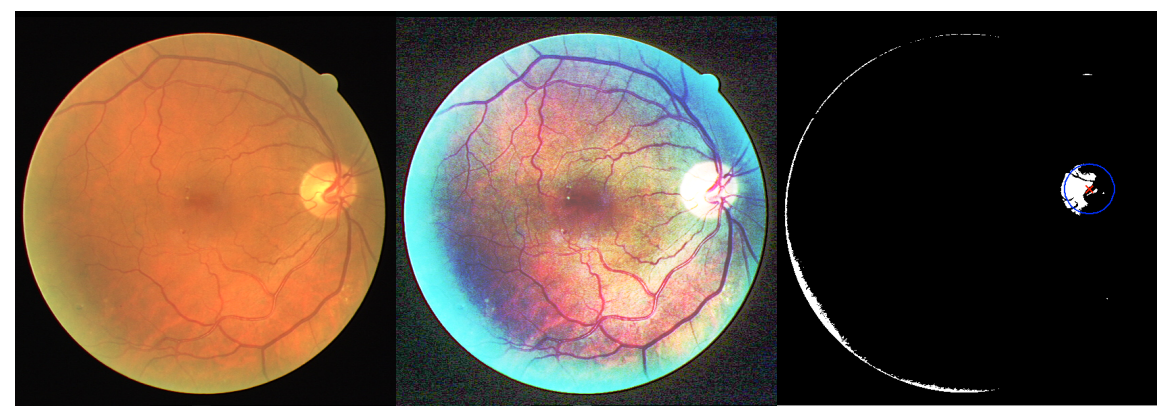

(a) Input image

(b) Histogram Equal

(c) $1 \%$ brightest pixels

Fig. 1. Finding the optical disc

results both in accuracy and detection time especially when testing robustness. In this case only few edges of the optical disc are detected making it often even impossible to locate it with the Hough transform.

So we adapted this technique by taking into account the fact that the optical disc is usually an object with the highest luminance values in the image. Another possible criterion suggested in literature is highest variance [11. In order to use a global threshold $t$ for all possible input images (and also distorted versions) we first apply a histogram equalization to the image. Using the highest 1 percent of the intensity image pixels $(t=0.99)$ a binary image is created that predominantly contains pixels of the optical disc (see Fig. 1. c). For finding the center of the circle that encloses the majority of pixels concentrated in a small region finally the Hough transform is applied locally. Fig. 1 displays the steps for finding the optical disc.

\subsection{Vessel Extraction}

Different approaches for automatic vessel segmentation have been proposed in literature (e.g. [7]). We have chosen to adapt the MATLAB software package mlvessel2 2 based on the wavelet-domain method described in [3] since it yields good results in enhancing vessel contrast while filtering out noise. First the retinal image is pre-processed by artificially extending the border that is defined by the camera's aperture in order to remove the strong contrast between the optical fundus and the image mask. Realizing that the wavelet transform is able to filter locally makes it effective for detecting local properties such as blood vessels. The continuous wavelet transform of a signal $f(x)$ is defined

$$
W_{\psi}(b, a)=\frac{1}{\sqrt{a}} \int f(x) \psi^{*}\left(\frac{x-b}{a}\right) d^{2} x
$$

$\overline{2}$ http://www .retina.iv.fapesp.br 
with $\psi^{*}$ denoting the complex conjugate of the 2-D Morlet wavelet $\psi$ defined by [3] as:

$$
\psi(x)=e^{i k_{0} x} e^{-\frac{1}{2}|A x|^{2}}
$$

where $A=\operatorname{diag}[\sqrt{\epsilon}, 1], \epsilon \geq 1$ is a $2 \times 2$ diagonal matrix defining the anisotropy of the filter. We only use the results produced by the Morlet wavelet with parameters $a=2, k_{0}=[0,3]$ and $\epsilon=4$ since this yields the best resolution of vessels. So for the scale value $a=2$ maximum response over all possible angles of the Morlet wavelet starting from 0 up to 170 degrees in steps of 10 is being calculated.

The resulting feature image is used for creating a binary vessel segmentation image by thresholding. Simple thresholding is the method of first choice because of its speed. However using a global threshold results in very different binary images concerning the number of vessels thus it is not very suitable for creating a retina template and matching. In order to achieve a well-balanced number of vessels in the binary images we first statistically determine the mean value of vessel pixels in a set of typical images suited for matching. For the data base used in our experiments (see Section 4.1) this gave us a mean value of vessel pixels of $8.5 \%$ and a standard deviation of 1,5\%. Starting from a standard threshold we slightly adjust the threshold up or down until the number of vessel pixels meets our above criteria. In order to get rid of unconnected vessels resulting from our thresholding process all connected objects that have fewer than a certain number of pixels are removed (see Fig. 2).

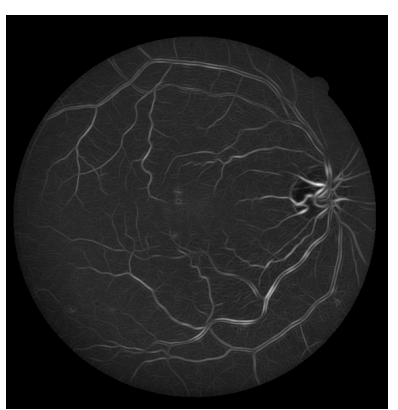

(a) Filtered image

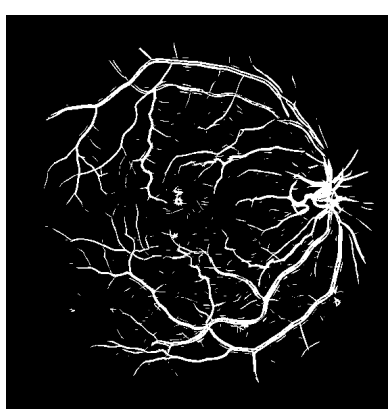

(b) Thresholding

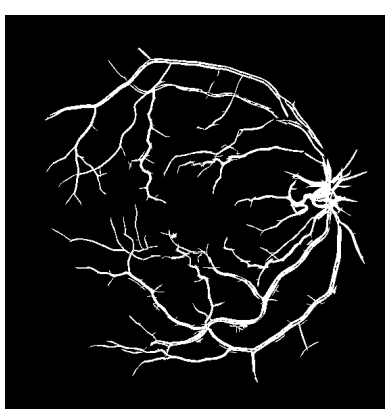

(c) Remove small objects

Fig. 2. Vessel Segmentation

\section{Retina Code Generation}

Starting from the center of the optical disc we use concentric circles for taking samples from the binary vessel images. For construction of these circles we have implemented Bresenham's circle drawing algorithm [1]. For every circle pixel its value is set according to the underlying vessel or non-vessel pixel. Then the values of $n_{\text {avg }}$ neighbouring circles are averaged and transformed to polar coordinates 
Table 1. Sampling settings

\begin{tabular}{ccccc}
\hline Name & $N_{\text {circ }}$ & $n_{\text {step }}$ & $n_{\text {avg }} r_{0}$ \\
\hline L1 & 15 & 3 & 3 & 5 \\
L2 & 30 & 3 & 3 & 5 \\
H1 & 60 & 5 & 3 & 5 \\
\hline
\end{tabular}

in steps of $1^{\circ}$. It is important to mention that outer circles usually degrade to circular arcs since the optical disc is mostly located at the left or right border of the retinal surface. This results in lower information density concerning the whole retina code which is further decreased by the higher arc length of the circles when using steps of $1^{\circ}$ and the fact that the density of the vessels is usually higher around the optical disc. Thus hitting an underlying vessel pixel becomes more unlikely for bigger (outer) circles. This is confirmed also in the example in Fig. 3. c. So we see that it is vital for our method to set the right parameters for the sampling procedure i.e., the number of samples (circles) $N_{c i r c}$, the averaging value $n_{\text {avg }}$ (i.e. how many neighbouring circles are used for producing a single bit value), and the radius of the first circle $r_{0}$.

We investigate different retina code variants as shown in the settings of Table 1 in order to see how the sampling parameters affect the matching performance. The resulting retina codes are of sizes 360x5 (225 Byte), 360x10 (450 Byte) and 360x20 (900 Byte). These templates can be further compressed by using Run-Length Encoding since there are usually long sequences of non-vessel pixels within each code. Retica Systems Inc. provide the information of using 50-100 or even 20-50 bytes for their templates, but this could refer to encoded data.

Setting L1 only samples around the optical disc (mostly resulting in entire circles), L2 is the same as L1 but with increased $N_{\text {circ }}$ and H1 also includes degraded circles due to the increased sampling area. Examples for the resulting retina codes from this sampling process are shown in Fig. 3 .

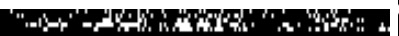

(a) Setting L1

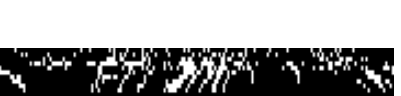

(b) Setting L2

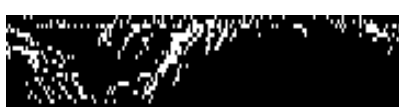

(c) Setting H1

Fig. 3. Retina Codes

\section{Experiments}

\subsection{Experimental Settings}

We tested and evaluated our methods on a publicly available database of nonmydriatic images and corresponding manual vessel segmentations: the DRIVE 3

\footnotetext{
${ }^{3}$ www.isi.uu.nl/Research/Databases/DRIVE/
} 
Table 2. Robustness Tests

\begin{tabular}{ll}
\hline Test & Settings \\
\hline JPEG & Quality $10 \%$ \\
JPEG2000 & Compression ratio $100: 1$ \\
Rotation & $90^{\circ},-90^{\circ}$ and $180^{\circ}$ \\
Sharpening & $\mathrm{r}=1$ pixel, $\sigma=1$, amount $=500 \%$ \\
Hist. Equal. Standard flat histogram \\
\hline
\end{tabular}

database [2]. The DRIVE database consists of 40 images that were captured in digital form from a Canon CR5 non-mydriatic 3CCD camera at 45 field of view (FOV). The images are stored in TIFF format of size 565 x 584 with 8 bits per color channel. Since the DRIVE database only contains images of different persons our experiments are limited to examining the inter person variability and FMR under image distortions. The green channel of the non-mydriatic images shows the best contrast so we chose it for optical disc detection as well as for vessel extraction (and subsequent code generation).

Matching between two distinct retina codes is done by calculating their Hamming distance. In order to compensate for rotated versions of the images the two retina patterns are shifted against each other and the minimum of all Hamming distances is calculated. For each image to be tested the Hamming distance with each of the remaining templates in the database is determined ("leave one out" strategy). A pair having Hamming distance below a decision threshold $T$ indicates a positive match.

For testing the robustness and the performance of our approach we generate several distorted versions of our input images by using the open-source tool Imagemagick (see Tab. 2 for the specifications) and matching the resulting templates with the images in the database.

\subsection{Experimental Results}

The first step in testing our method is matching all retina codes against each other (for each of the settings shown in Tab1) to see how the scores are distributed and if the codes are sufficiently discriminative. The mean relative Hamming distances $\bar{h}$, their standard deviations $s_{h}$, and the maximum and minimum Hamming distances $h_{\max }$ and $h_{\min }$ for this test are shown in Tab. 3, Assuming uncorrelated templates from different persons an average close to 0.5 in terms of Hamming distance is expected. In fact, the mean Hamming distances $\bar{h}$ are much smaller $(0.123 \leq \bar{h} \leq 0.216)$. In addition to that, the range of obtained Hamming distances $\left[h_{\min }, h_{\max }\right]$ is very small and covers only $7-8 \%$ of the overall possible range.

Setting L2 shows the best results with respect to highest average Hamming distance values and standard deviation. The low values for $\mathrm{H} 1$ may be explained when taking Fig 3. c as example: of course, the large black areas - stemming from the circular arcs without any vessels close to the images' edges - in the 
Table 3. Score distribution

\begin{tabular}{ccccc}
\hline & $\bar{h}$ & $s_{h}$ & $h_{\max }$ & $h_{\min }$ \\
\hline L1 & 0.206 & 0.022 & 0.236 & 0.165 \\
L2 & 0.216 & 0.028 & 0.250 & 0.172 \\
H1 & 0.123 & 0.027 & 0.172 & 0.079 \\
\hline
\end{tabular}

Table 4. Results of Robustness Tests

\begin{tabular}{|c|c|c|c|c|c|c|c|c|c|}
\hline & \multicolumn{3}{|c|}{ L1 } & \multicolumn{3}{|c|}{$\mathrm{L} 2$} & \multicolumn{3}{|c|}{ H1 } \\
\hline & $h_{\max }$ & $h_{\min }$ & $F M R$ & $h_{\max }$ & $\left|h_{\min }\right|$ & $F M R$ & $h_{\max }$ & $\left|h_{\min }\right|$ & $F M R$ \\
\hline JPEG & 0.115 & 0.053 & $0 \%$ & $|0.107|$ & $|0.064|$ & $0 \%$ & 0.074 & 0.032 & $0 \%$ \\
\hline JPEG2000 & 0.108 & 0.065 & $0 \%$ & 0.109 & 0.057 & $0 \%$ & 0.104 & 0.037 & $25 \%$ \\
\hline Rotation & 0.151 & 0.073 & $0 \%$ & 0.115 & 0.081 & $0 \%$ & 0.095 & 0.039 & $25 \%$ \\
\hline Hist. Equal. & 0.172 & 0.097 & $17.5 \%$ & 0.187 & 0.119 & $5 \%$ & 0.118 & 0.055 & $17.5 \%$ \\
\hline Sharpening & 0.181 & 0.121 & $2.5 \%$ & 0.158 & 0.124 & $0 \%$ & 0.103 & 0.06 & $30 \%$ \\
\hline
\end{tabular}

code result in low Hamming distances. This is also true (in less pronounced manner) for the other settings where we also find an imbalance between black (non-vessel) and white (vessel) areas causing low differences in general. These results indicate a very low inter personal variability of the generated code which makes the occurrence of false positive matches highly probable. While actual matching performance can not be derived directly from these values since intra personal variability can not be assessed at present state (due to the lack of corresponding data in the DRIVE database), low inter personal variability suggests the approach not to be suited for larger populations at least. The retina code example given by Retica Systems Inc. ("Multi-Radius Digital Pattern"4) seems to indicate even smaller potential for high variability (since the generation is not explained in detail, a reliable statement on this issue is not possible of course). Recall that Retica Systems Inc. claims a template size of 20 - 100 bytes whereas the smallest template investigated here has 225 byte. This of course worsens the situation for the commercial system. Also, the comparison to an iris code5 suggests the retina code to be of significantly lower variability potential.

The results of the robustness test are shown in Tab. 4. Again, $h_{\max }$ denotes the highest relative Hamming distance of all matches, $h_{\text {min }}$ the lowest value and $F M R$ indicates the FMR ratio (ratio of false positive matches and the number of tests performed). The decision threshold $T$ for computing FMR was derived from the score distribution test and is set to $T=h_{\min }$ for all subsequent robustness and sampling tests (see Tab. 3).

Setting L2 performs best of all having only minor problems when the image is histogram-equalized. This is usually a problem with vessel segmentation yielding

\footnotetext{
${ }^{4}$ http://www.retica.com/site/images/howitworks.pdf

${ }^{5}$ http://www.retica.com/site/technology/irisretina.html
} 
to many vessels and sometimes distorting the code too much. Both Settings L1 and L2 show very good robustness against false positive matches even under severe compression. This confirms previous results on lossy compression of biometric sample data not to effect FAR as long as applied in sensible ranges. Also rotation and sharpening does not lead to false positives in case of L2. A severe problem occurs with lowpass filtering. Here the vessels lying over the optical disc cannot be clearly distinguished any more, resulting in corrupted codes for all settings (since sampling near the optical disc is most crucial for our templates). Thus Lowpass filtering is omitted in Tab. 4. H1 shows very poor results with respect to robustness. Even rotation leads to $25 \%$ FMR although rotation is compensated in the matching stage. It is also remarkable that the higher sampling rate of $\mathrm{H} 1$ does not at all improve accuracy when using distorted images. Overall, it gets clear that the sampling strategy for $\mathrm{H} 1$ is not at all suited for generating sensible retina codes.

\section{Conclusion and Future Work}

The methods considered for generating retina codes from retinal images have exhibited very low inter personal variability - given the fact that retina based biometrics are probably restricted to high security environments due to the inconvenient data acquisition process, the real-life applicability of these techniques as a stand-alone technique is as least questionable (as long as the encoding of the data is not adapted properly). Good robustness against JPEG and JPEG2000 compression not leading to false positives at low bitrates has been observed. Note that all those findings only apply to retina code templates but not for retina-scan based biometrics in general.

Future work will involve a cooperation with the Department of Ophthalmology at the local hospital to get access to data allowing the determination of intra personal variability and to study the effects of eye diseases on a retina code based recognition scheme. Additionally, we will study the effect of using runlength encoding of the retina codes on the resulting Hamming distances.

\section{References}

1. Bresenham, J.: A linear algorithm for incremental display of circular arcs. Communications of the ACM 20(2), 100-106 (1977)

2. Staal, J., Abramoff, M., Niemeijer, M., Viergever, M., van Ginneken, B.: Ridge based vessel segmentation in color images of the retina. IEEE Transactions on Medical Imaging 23(4), 501-509 (2004)

3. Soares, J.V.B., Leandro, J.J.G., Cesar Jr., R.M., Jelinek, H.F., Cree, M.J.: Retinal vessel segmentation using the 2 -d gabor wavelet and supervised classification. IEEE Transactions on Medical Imaging 25(9), 1214-1222 (2006)

4. Nanavati, S., Thieme, M., Nanavati, R.: Biometrics - Identity verification in a networked world. Wiley Computer Publishing, Chichester (2002)

5. Daugman, J.: How iris recognition works. IEEE Transactions on Circiuts and Systems for Video Technology 14(1), 21-30 (2004) 
6. Ives, R., Guidry, A., Etter, D.: Iris recognition using histogram analysis. In: Conference Record of the 38th Asilomar Conference on Signals, Systems, and Computers, vol. 1, pp. 562-566. IEEE Signal Processing Society, Los Alamitos (2004)

7. Vermeer, K., Vos, F., Lemij, H., Vossepoel, A.: A model based method for retinal blood vessel detection. Computers in Biology and Medicine 34, 209-219 (2004)

8. Lin, T., Zheng, Y.: Node-matching-based pattern recognition method for retinal blood vessel images. Optical Engineering 42(11), 3302-3306 (2003)

9. Xu, Z., Guo, X., Hu, X., Chen, X., Wang, Z.: The identification and recognition based on point for blood vessel of ocular fundus. In: Zhang, D., Jain, A.K. (eds.) ICB 2005. LNCS, vol. 3832, pp. 770-776. Springer, Heidelberg (2005)

10. Barrett, S.F., Naess, E., Molvik, T.: Employing the hough transform to locate the optic disk. Biomedical Sciences Instrumentation 37, 81-86 (2001)

11. Sinthanayothin, C., Boyce, J., Cook, H., Williamson, T.: Automated localisation of the optic disc, fovea, and retinal blood vessels from digital colour fundus images. British Journal of Ophthalmology 83, 902-910 (1999) 